\title{
Correction to: A Practical Optimal Guidance Scheme Under Impact Angle and Terminal Acceleration Constraints
}

\author{
Hyo-Seon $\mathrm{Chi}^{1} \cdot$ Yong-In Lee ${ }^{2} \cdot$ Chang-Hun Lee ${ }^{1} \cdot$ Han-Lim Choi ${ }^{1}$ \\ (c) The Korean Society for Aeronautical \& Space Sciences 2021

\section{Correction to: \\ International Journal of Aeronautical and Space Sciences \\ (2021) 22:923-935 \\ https://doi.org/10.1007/s42405-020-00339-7}

Due to an unfortunate mistake during the correction process, Fig. 7 overlaps the text.

The text on that page should read:

OGL-IATA $/ 1$ with $\alpha=1.7 \mathrm{rad} / \mathrm{s}$ is applied to the high-order missile dynamics as shown in Eq. (40) with PAF or without PAF.

First, the stability of the guidance and control loop is analyzed in both cases. To obtain the open-loop transfer function of the guidance and control loop, we disconnect a signal between the guidance module and the control module as shown in Fig. 9. Since the characteristic of the guidance and control loop is different depending on the remaining time of interception (i.e., the time-to-go), the stability analysis is performed for different time-to-go values by utilizing the freezing method. The stability of the guidance and control loop is then measured by the vector margin [32].

Nonlinear simulation conditions, according to the feedback acceleration types, are described in Table 2. Case 1 is the OGL-IATA/1 without PAF and Case 2 denotes the

The original article can be found online at https://doi.org/10.1007/ s42405-020-00339-7.

\footnotetext{
Han-Lim Choi

hanlimc@kaist.ac.kr

Hyo-Seon Chi

hschi@lics.kaist.ac.kr

Yong-In Lee

yilee@add.re.kr

Chang-Hun Lee

lckdgns@kaist.ac.kr

1 Department of Aerospace Engineering, Korea Advanced Institute of Science and Technology (KAIST), 291

Daehak-ro, Yuseong-gu, Daejeon 34141, Korea

2 Agency for Defense Development (ADD), Yuseong, P. O. Box 35, Daejeon 34186, Korea
}

OGL-IATA/1 with PAF calculated by Eq. (40). Case 3 is the OGL-IATA/1 with PAF/1, first-order approximated dynamics used in the OGL-IATA/1.

Figure 10a represents the Nyquist plot for the two cases at $t_{\mathrm{go}}=2 \mathrm{~s}$ as an illustrative example. The OGL-IATA/1 with $\mathrm{PAF} / 1$ case focuses on the stability of the guidance loop and simplifies time-varying dynamics, making it easy to apply in practice. In these cases, the gain margin and the phase margin are recorded as provided in Table 3 . The results obtained indicate that PAF improves the stability of the guidance and control loop when implementing OGL-IATA/1. Figure 10b shows the pattern of the vector margin according to changes in the freezing time-to-go values. As shown in Fig. 10b, the vector margin of the OGL-IATA/1 with PAF is greater than that of the OGL-IATAl1 without PAF. This tendency is more pronounced when the remaining time of interception is short. Since the instability of the guidance and control loop directly affects the guidance performance at the moment of interception, the above characteristic is desirable to ensure some robustness for the guidance operations.

Figure 11 shows the nonlinear simulation results when OGL-IATA/1 with/without PAF is applied to the high-order missile dynamics with uncertain time delay. In this simulation, the time delay of $0.1 \mathrm{~s}$ is considered to investigate the robustness of OGL-IATA/1 with/without PAF against the time delay in the guidance loop. Additionally, the acceleration limit is not considered to observe the pattern of acceleration divergence at the moment of interception. Figure 11a-c represents the trajectory, the flight path angles, and the accelerations, respectively. In all cases, terminal acceleration errors are observed. However, the results obtained indicate that the acceleration divergence is 
Figure 7 should be read:

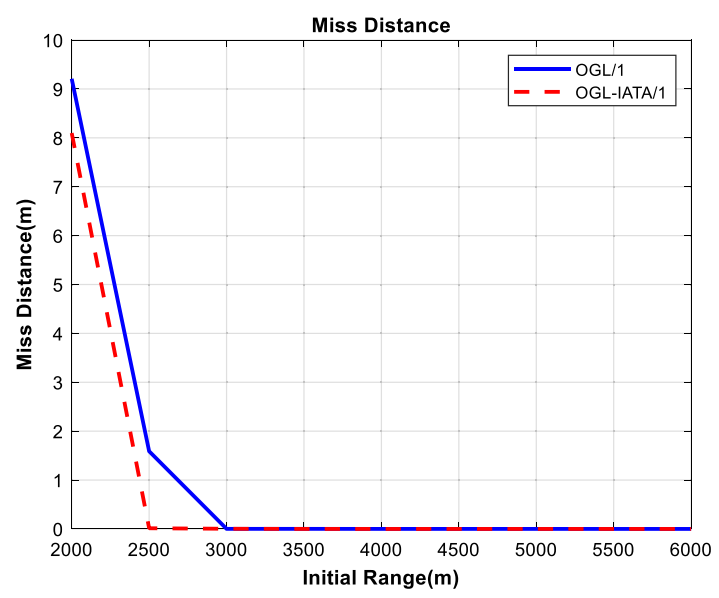

(a) Miss distances

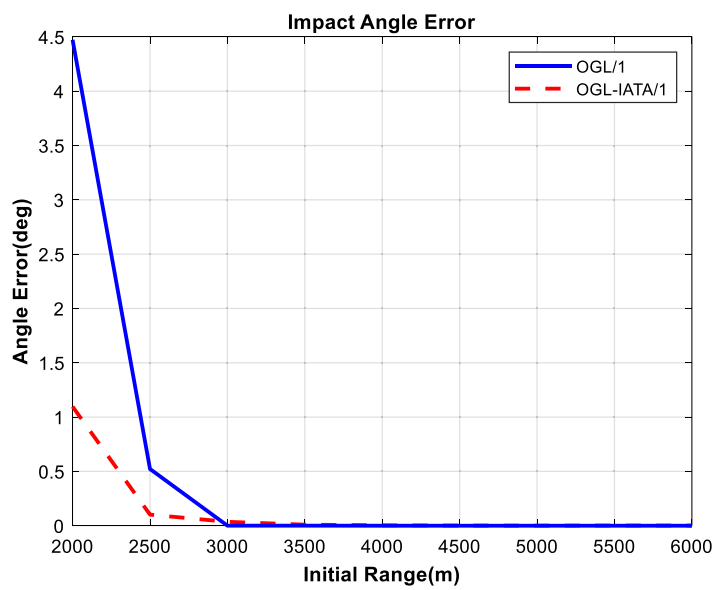

(b) Impact Angle Errors

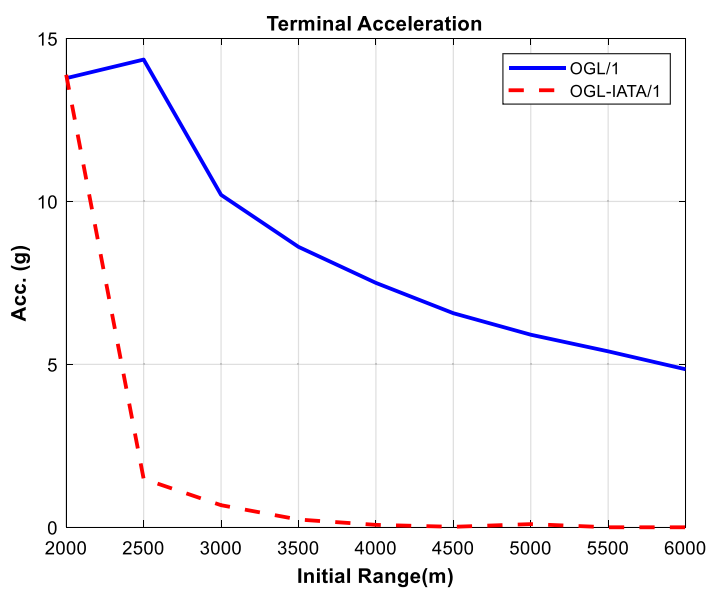

(c) Terminal Accelerations

Fig. 7 The terminal error analyses for various initial ranges under the OGL-IATA/1 and OGL/1. a Miss distances, b impact angle errors, c terminal accelerations
The original article has been corrected.

Publisher's Note Springer Nature remains neutral with regard to jurisdictional claims in published maps and institutional affiliations. 